\title{
Avaliação das Propriedades Mecânicas e da Permeabilidade a Gases de Membranas Obtidas a Partir de Dispersões Aquosas de Poliuretanos à Base de Polibutadieno Líquido Hidroxilado
}

\author{
Fernanda M. B. Coutinho, Marcia C. Delpech \\ Instituto de Química, UERJ \\ Maria Elizabeth F. Garcia \\ COPPE, UFRJ
}

Resumo: O desenvolvimento de sistemas não-poluentes tem sido cada vez mais necessário para atender às exigências ambientais. Neste trabalho foram sintetizadas formulações de poliuretanos à base de água para a obtenção de membranas densas cujas propriedades mecânicas e de permeação a $\mathrm{CO}_{2}$ e $\mathrm{N}_{2}$ foram avaliadas. Foram empregados como monômeros polibutadieno líquido hidroxilado (HTPB), poli(glicol propilênico) (PPG), diisocianato de isoforona (IPDI) e ácido dimetilolpropiônico (DMPA). Os grupos carboxílicos foram neutralizados com trietilamina (TEA) e o extensor de cadeia utilizado foi a etilenodiamina (EDA). As membranas foram obtidas como filmes vazados a partir das dispersões aquosas. Os resultados mostraram que o aumento no teor de HTPB na formulação levou a uma maior resistência mecânica bem como a um aumento na permeabilidade e na seletividade das membranas a $\mathrm{CO}_{2}$.

Palavras-chave: Poliuretanos, dispersões aquosas, membranas densas, permeabilidade a gases, seletividade.

\section{Evaluation of Mechanical Properties and Gas Permeability of Membranes Obtained from Polyurethane Aqueous Dispersions Based on Hydroxyl-Terminated Polybutadiene}

\begin{abstract}
The performance of dense membranes based on nonpolluting systems of polyurethane aqueous dispersions was evaluated. The mechanical properties and the selectivity and permeability to $\mathrm{CO}_{2}$ and $\mathrm{N}_{2}$ were determined. The aqueous systems were based on hydroxyl-terminated polybutadiene (HTPB), poly(propylene glycol) (PPG), isophorone diisocyanate (IPDI), and dimethylolpropionic acid (DMPA). The membranes obtained from cast films provided higher permeability and selectivity to $\mathrm{CO}_{2}$ as the HTPB content increased. The tensile strength and the modulus values of the materials increased and the elongation decreased with higher amounts of HTPB. As a result, the presence of HTPB improved the mechanical resistance, the permeability and the selectivity to $\mathrm{CO}_{2}$ of the polyurethane membranes.
\end{abstract}

Keywords: Polyurethanes, aqueous dispersions, dense membranes, gas permeability, selectivity.

\section{Introdução}

O desenvolvimento de sistemas não poluentes está sendo cada vez mais incentivado por questões ambientais e, dentre eles, destacam-se as dispersões aquosas de poliuretanos. A versatilidade estrutural desses polímeros se deve à possibilidade de escolha de uma ampla gama de diisocianatos e polióis como monômeros ${ }^{[1]}$. Dentre os polióis destacam-se, tanto em trabalhos acadêmicos quanto em produção industrial, os poliéteres e os poliésteres. $\mathrm{O}$ polibutadieno líquido hidroxilado (HTPB), no entanto, é empregado de forma restrita, devido à sua natureza hidrofóbica, que dificulta bastante a dispersão em água ${ }^{[2]}$.

Poliuretanos à base de HTPB, geralmente obtidos em meio orgânico, são muito utilizados na fabricação de adesivos, revestimentos, elastômeros, propelentes e agentes encapsulantes em diversos campos, como na indústria automobilística, aeroespacial, de construção e eletrônica ${ }^{[3-4]}$. $\mathrm{Na}$ área médica, esses polímeros são muito empregados devido à sua natureza biocompatíve ${ }^{[5]}$. Outra importante área de aplicação desses materiais é na fabricação de membranas para separação de gases ${ }^{[6-7]}$.

A permeação de gases em materiais não-porosos, como polímeros, tem sido extensivamente estudada devido a sua importância tecnológica em aplicações industriais, tanto na área de revestimentos quanto na de separação de gases por membranas ${ }^{[8]}$. Uma importante aplicação dos poliuretanos é no desenvolvimento dessas membranas que, além de

Autor para correspondência: Fernanda M. B. Coutinho, Departamento de Processos Químicos, Instituto de Química, UERJ, PHLC, Rua São Francisco Xavier 524 - Maracanã, CEP: 20559-900, Rio de Janeiro, RJ. E-mail: fern@uerj.br 
separarem efetivamente misturas de gases, como $\mathrm{CO}_{2}$ e $\mathrm{N}_{2}$, podem ser utilizadas em recuperação de $\mathrm{CO}_{2}$ do petróleo, remoção de $\mathrm{He}$ do gás natural e recuperação de $\mathrm{H}_{2}$ da corrente de gás de purga de plantas de amônia, dentre outras ${ }^{[9-10]}$.

As principais propriedades vinculadas a membranas para separação de gases são alta capacidade de permeação, estabilidade térmica, resistência química e resistência mecânica ${ }^{[11]}$. Poliuretanos à base de HTPB são especialmente adequados no emprego como membranas tal fim devido à flexibilidade desses materiais em baixas temperaturas e à alta segregação de fases entre os domínios rígidos e flexíveis formados pelas cadeias $^{[7]}$.

Certamente, é de grande interesse científico e tecnológico o desenvolvimento de resinas não-poluentes para a manufatura de sistemas para separação de gases. O objetivo deste estudo foi a obtenção de membranas densas a partir de dispersões aquosas de poliuretanos à base de HTPB, poli(glicol proprilênico) (PPG), ácido dimetilolpropiônico (DMPA) e diisocianato de isoforona (IPDI). Essas formulações e similares, obtidas como emulsões aquosas e empregadas como membranas para a separação de gases, não foram relatadas por outros grupos de pesquisa.

O objetivo geral deste trabalho foi, portanto, a avaliação da resistência mecânica, bem como das propriedades de permeabilidade e de seletividade a $\mathrm{CO}_{2}$ e $\mathrm{N}_{2}$, de membranas obtidas a partir de resinas não-poluentes.

\section{Experimental}

\section{Materiais}

Os seguintes reagentes, empregados na síntese das membranas, foram usados como recebidos: ácido dimetilolpropiônico (DMPA), Aldrich; dibutildilaurato de estanho (DBTDL), Aldrich; diisocianato de isoforona (IPDI), Hülls; etilenodiamina (EDA), Reagen; polibutadieno líquido hidroxilado (HTPB) $\left(\overline{\mathrm{M}}_{\mathrm{n}}=3500 \mathrm{~g} / \mathrm{mol}\right.$, determinado por osmometria de pressão de vapor (VPO), em tolueno a $50{ }^{\circ} \mathrm{C}$ ), Petroflex; poli(glicol propilênico) (PPG) $\left(\overline{\mathrm{M}}_{\mathrm{v}}=2500 \mathrm{~g} / \mathrm{mol}\right.$, determinado em tolueno, a $20{ }^{\circ} \mathrm{C}$ ), Dow Chemical; e trietilamina (TEA), Union Carbide.

\section{Métodos}

$\mathrm{Na}$ síntese dos poliuretanos, inicialmente foi preparado o pré-polímero com HTPB, PPG, DMPA e IPDI. A reação foi catalisada por DBTD. Os grupos carboxílicos provenientes do DMPA foram neutralizados com TEA. A massa reacional foi então submetida às etapas de dispersão em água e extensão de cadeia com EDA. Nessa última etapa, houve a formação de ligações uréia na cadeia ${ }^{[11-13]}$. Foram sintetizados pré-polímeros com $5 \%$ em massa de DMPA em relação à massa total.

A razão entre o número de equivalentes-grama de grupos isocianato e hidroxila $(\mathrm{NCO} / \mathrm{OH})$ foi variada em dois valores extremos: 1,5 e 3,0. Observou-se que, para valores inferiores a 1,5 , não foi possível dispersar o pré-polímero em água devido à alta viscosidade do meio. A razão $\mathrm{NCO} / \mathrm{OH}=1,5$ foi, portanto, o valor mínimo encontrado para a produção das dispersões. A razão $\mathrm{NCO} / \mathrm{OH}=3,0$ foi limite para a obtenção de membranas íntegras e homogêneas. Razões superiores forneceram filmes quebradiços, não adequados para a manufatura de membranas.

Nas formulações poliuretânicas, também foi variado o percentual em massa de HTPB, em função da massa total de pré-polímero. Para a razão $\mathrm{NCO} / \mathrm{OH}=1,5$, a faixa variou de 0 a $50 \%$. Teores de HTPB superiores a 50\% impediam a dispersão pois levavam à formação de pré-polímeros com altíssima viscosidade. A razão $\mathrm{NCO} / \mathrm{OH}$, foi variada de 0 a $10 \%$, pois razões mais altas levaram à formação de membranas rígidas e quebradiças.

As dispersões aquosas foram vazadas em placas de teflon niveladas e secas ao ar por 7 dias e a $60^{\circ} \mathrm{C}$ em estufa, por 12 horas. Foram obtidos filmes, com espessuras variando na faixa de 60 a $100 \mu \mathrm{m}$, que deram origem a membranas densas, de forma arredondada, e a corpos de prova, cortados de forma retangular $(5 \mathrm{~mm} \times 70 \mathrm{~mm})$, que foram empregados nos ensaios mecânicos.

A avaliação das propriedades mecânicas das membranas foi feita em Máquina Universal de Ensaios Instron 4202, com garras pneumáticas, com velocidade de separação de $500 \mathrm{~mm} / \mathrm{min}$, e célula de $1 \mathrm{kN}$, segundo método ASTM adaptado ${ }^{[12-13]}$.

O equipamento de permeação utilizado na caracterização das membranas foi desenvolvido no laboratórios da COPPE/ UFRJ e é ilustrado na Figura 1. Consiste de uma célula de permeação em aço inox (A) com área de $7,07 \mathrm{~cm}^{2}$, formada por dois compartimentos separados pela membrana: o superior, por onde é alimentado o gás puro (B) a uma pressão de 3 bar, e o inferior, que é conectado a um transdutor de pressão (C), que por sua vez está ligado a um miliamperímetro (D). A vazão de gás é determinada medindo-se o tempo gasto para acumular certo volume de gás no transdutor, que gera um sinal no miliamperímetro, previamente calibrado. A quantidade de gás permeado é calculada utilizando-se a lei dos gases ideais, uma vez que o volume do sistema foi previamente medido, sendo igual a $18,24 \mathrm{~cm}^{3}$.

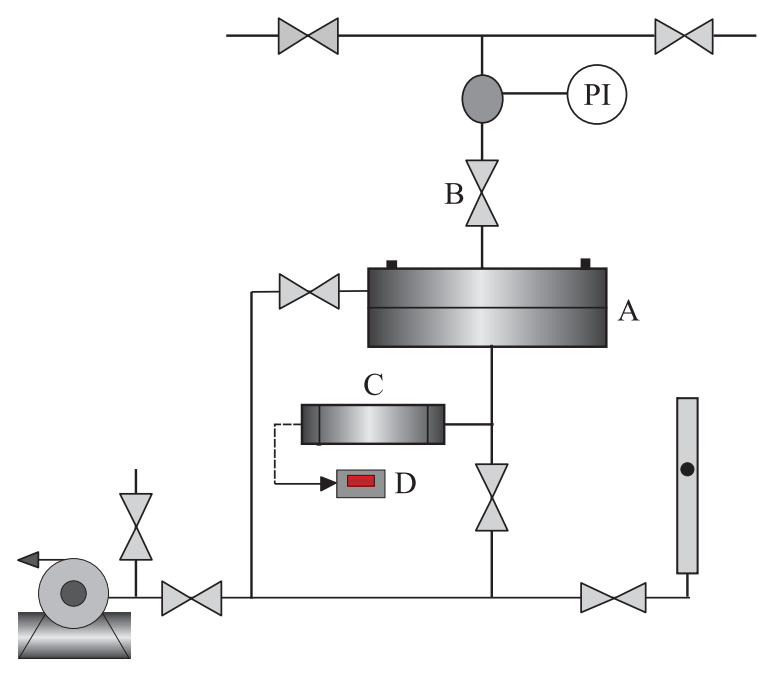

Figura 1. Representação esquemática do sistema de permeação de gás 
Os valores de permeabilidade dos gases $\mathrm{CO}_{2},\left(\mathrm{P} / 1_{\mathrm{CO}_{2}}\right) \mathrm{e}$ $\mathrm{N}_{2}\left(\mathrm{P} / \mathrm{N}_{2}\right)$, no estado estacionário, foram calculados segundo a Equação 1 e a razão entre ambos forneceu a seletividade (Equação 2).

No sistema de permeação, foi determinado o sinal fornecido pelo miliamperímetro em função do tempo em segundos. A partir da curva de calibração do sistema, a qual forneceu o valor do sinal em função da diferença de pressão aplicada, podese determinar a curva de pressão em função do tempo e, assim, o valor de dp/dt (coeficiente angular da curva) para cada membrana. O volume e a área da célula eram fixos e conhecidos, e os demais parâmetros foram facilmente mensuráveis. Desta forma a permeabilidade por unidade de espessura pode ser determinada para um determinado valor de $\Delta \mathrm{p}$. As permeabilidades foram determinadas para cada membrana e normalizadas para uma mesma espessura.

$$
\frac{\mathrm{P}}{l}=\frac{\mathrm{dp}}{\mathrm{dt}} \cdot\left(\frac{\mathrm{V}_{\text {sistema }}}{\mathrm{A} \Delta \mathrm{p}}\right) \cdot\left(\frac{\mathrm{TCNTP}}{\text { TAmbiente. }_{\mathrm{CNTP}}}\right)
$$

onde:

$\mathrm{P} / 1=$ permeabilidade por unidade de espessura;

$I=$ espessura da membrana;

$\mathrm{V}=$ volume do sistema;

$\mathrm{A}=$ área de permeação;

$\Delta \mathrm{p}=$ diferença de pressão aplicada entre os dois lados da membrana;

$\mathrm{T}=$ temperatura do teste;

T e p $(\mathrm{CNTP})=$ temperatura e pressão absolutas.

$$
\alpha \mathrm{CO}_{2} / \mathrm{N}_{2}=P / l_{\mathrm{CO}_{2}} / P / l_{N_{2}}
$$

onde:

$\alpha_{\mathrm{CO}_{2}} / \mathrm{N}_{2}=$ seletividade

\section{Resultados e Discussão}

As dispersões aquosas, que deram origem às membranas densas, apresentaram viscosidade aparente na faixa de 30 a $220 \mathrm{cP}$, sendo que os limites superiores corresponderam às dispersões com maiores teores de HTPB, devido à maior viscosidade do meio reacional durante a dispersão em água. Todas as dispersões apresentaram estabilidade à sedimentação de partículas dispersas em períodos de observação superiores a seis meses.

As membranas preparadas eram formadas por poliuretanos termoplásticos (TPU), que podem ser considerados como copolímeros segmentados, ou multiblocos, do tipo (A-B) , consistindo de seqüências alternadas de segmentos rígidos e flexíveis ${ }^{[7]}$. Essa estrutura mista resulta na formação de microdomínios e conseqüente separação de fases, que é função do grau de miscibilidade entre os dois tipos de segmentos ${ }^{[1,13]}$.

Os segmentos rígidos ou duros, provenientes normalmente da reação do diisocianato com o extensor de cadeia, apresentam características semi-cristalinas, interagindo fortemente por ligações hidrogênio, provenientes dos grupamentos uretânicos. Essa associação provoca a formação de retículos físicos que são responsáveis pela estabilidade dimensional do poliuretano ${ }^{[1,13-17]}$.
Nas formulações estudadas, os segmentos rígidos resultaram da reação entre os grupos isocianato do IPDI e os grupos $\mathrm{NH}_{2}$ do extensor de cadeia EDA, que levou à formação de grupos ureicos, intensificando essas interações. $\mathrm{O}$ teor de segmentos rígidos é determinado pela razão $\mathrm{NCO} / \mathrm{OH}$. Um aumento nessa razão leva a um aumento na rigidez das cadeias.

Os segmentos flexíveis, que apresentam características borrachosas, conferem as propriedades elastoméricas às cadeias uretânicas e são formados comumente por polióis de massa molecular entre 600 e $4000 \mathrm{~g} / \mathrm{mol}^{[1,13-17]}$. Nas formulações sintetizadas, esses segmentos eram formados por HTPB e PPG. O DMPA, diol de massa molecular baixa, também foi incorporado à cadeia para possibilitar a dispersão em água, após a neutralização com TEA de seus grupos carboxílicos pendentes na cadeia poliuretânica.

As membranas obtidas eram transparentes e flexíveis, flexibilidade essa que diminuía visivelmente à medida que o teor de HTPB e a razão $\mathrm{NCO} / \mathrm{OH}$ aumentavam na formulação. As membranas sintetizadas a partir desse poliol também apresentaram uma tonalidade amarelada que se intensificava à medida que seu teor aumentava no material, principalmente para as amostras sintetizadas com razão $\mathrm{NCO} / \mathrm{OH}=1,5$, nas quais o teor de segmentos flexíveis era mais alto. Essa característica é decorrente da presença de hidrogênios alílicos na cadeia do HTPB, suscetíveis a processos termooxidativos ${ }^{[11]}$. Quando os segmentos flexíveis são compostos por HTPB, em contrapartida à presença de poliéteres como o PPG, ocorre uma maior segregação entre os domínios rígidos e flexíveis devido ao caráter apolar que a cadeia de polibutadieno confere ao poliuretano. Como conseqüência, maior resistência à hidrólise, redução na permeabilidade à água e aumento na resistência mecânica são propriedades incorporadas ao material ${ }^{[6,14,18,19]}$.

A Tabela 1 apresenta os dados obtidos para as propriedades mecânicas (tensão na ruptura, módulo de elasticidade e alongamento na ruptura) e para a permeabilidade e seletividade das membranas a $\mathrm{CO}_{2}$ e $\mathrm{N}_{2}$.

Comparando-se as membranas obtidas a partir da razão 1,5, pode-se observar que a amostra contendo $0 \%$ de HTPB em sua formulação apresentou um elevado valor de alongamento na ruptura, ou seja, alta elasticidade. No entanto a resistência mecânica foi muito reduzida, observando-se os baixos valores obtidos para o módulo de elasticidade e para a tensão na ruptura. Em contrapartida, a presença de uma pequena proporção de HTPB na formulação $(10 \%)$ já aumentou significativamente o valor da tensão na ruptura, o que indica uma melhora expressiva na resistência mecânica do material. No entanto, foi observado que teores superiores a $10 \%$ de HTPB na formulação provocaram apenas uma ligeira elevação na tensão na ruptura. Já o módulo de elasticidade aumentou significativamente na formulação para o teor mais alto de HTPB $(50 \%)$.

$\mathrm{O}$ alongamento na ruptura diminuiu drasticamente com a presença de HTPB (10\%) na formulação, em relação à amostra na qual esse poliol esteve ausente. Essa redução foi pouco expressiva para os teores $20 \%$ e $30 \%$ mas foi bastante significativa para o maior teor $(50 \%)$. 
Tabela 1. Propriedades mecânicas, permeabilidade a $\mathrm{CO}_{2}$ e $\mathrm{N}_{2}$ e seletividade de membranas à base de poliuretanos obtidos a partir de dispersões aquosas

\begin{tabular}{|c|c|c|c|c|c|c|}
\hline Membrana* & $\begin{array}{c}\varepsilon \\
(\%)\end{array}$ & $\begin{array}{c}\mathbf{E} \\
(\mathbf{M P a})\end{array}$ & $\begin{array}{c}\sigma \\
(\mathbf{M P a})\end{array}$ & $\begin{array}{c}\mathrm{P} / \mathrm{lCo} \mathrm{C}_{2} \\
{\left[\mathrm{~cm}^{3}(\mathrm{CNTP}) / \mathrm{cm}^{2} . \mathrm{s} . \mathrm{cm} \mathrm{Hg}\right]}\end{array}$ & $\begin{array}{c}\mathrm{P} / \mathrm{lN} \mathrm{N}_{2} \\
{\left[\mathrm{~cm}^{3}(\mathrm{CNTP}) / \mathrm{cm}^{2} . \mathrm{s} . \mathrm{cm} \mathrm{Hg}\right]}\end{array}$ & $\alpha \mathrm{CO}_{2} / \mathrm{N}_{2}$ \\
\hline \multicolumn{7}{|c|}{ Razão NCO/OH = 1,5 } \\
\hline 0 & 1461 & 0,7 & 0,5 & $0,25 \times 10^{-7}$ & $0,08 \times 10^{-8}$ & 31,3 \\
\hline 10 & 979 & 1 & 7 & $5,84 \times 10^{-7}$ & $5,57 \times 10^{-8}$ & 10,5 \\
\hline 20 & 902 & 3 & 9 & $7,93 \times 10^{-7}$ & $6,96 \times 10^{-8}$ & 11,4 \\
\hline 30 & 788 & 3 & 10 & $10,3 \times 10^{-7}$ & $6,96 \times 10^{-8}$ & 14,8 \\
\hline 50 & 509 & 10 & 10 & $15,7 \times 10^{-7}$ & $6,96 \times 10^{-8}$ & 22,6 \\
\hline \multicolumn{7}{|c|}{ Razão NCO/OH = 3,0 } \\
\hline 0 & 493 & 68 & 23 & $0,02 \times 10^{-7}$ & $0,08 \times 10^{-8}$ & 2,5 \\
\hline 10 & 471 & 142 & 24 & $1,5 \times 10^{-7}$ & $4,17 \times 10^{-8}$ & 3,6 \\
\hline
\end{tabular}

* Teor (\%) de HTPB em relação à massa total de pré-polímero

Parâmetros mecânicos: $\varepsilon=$ alongamento na ruptura; $\mathrm{E}=$ módulo de elasticidade; $\sigma=$ tensão na ruptura

Avaliação das membranas: $\mathrm{P} / 1=$ permeabilidade; $\alpha_{\mathrm{CO}_{2} / \mathrm{N}_{2}}=$ seletividade

As amostras obtidas com razão $\mathrm{NCO} / \mathrm{OH}=3,0$, com maior teor de segmentos rígidos, apresentaram interações mais intensas por ligação hidrogênio. A resistência mecânica dessas amostras teve um aumento marcante em relação às preparadas com razão $\mathrm{NCO} / \mathrm{OH}=1,5$, apresentando valores de módulo de elasticidade e tensão na ruptura significativamente maiores. Comparando-se as duas amostras obtidas com razão $\mathrm{NCO} / \mathrm{OH}=3,0$, pode-se observar que o HTPB conferiu maior resistência mecânica, expressa principalmente no valor do módulo. Os valores do alongamento na ruptura das amostras preparadas com razão $\mathrm{NCO} / \mathrm{OH}=3,0$ foram mais baixos do que aqueles observados para as obtidas com razão $\mathrm{NCO} / \mathrm{OH}=1,5$, mas sua redução não foi tão expressiva, mostrando que as membranas conservaram certa elasticidade.

Esses resultados sugerem que o HTPB aumenta a resistência mecânica do material, diminuindo sua elasticidade e, conseqüentemente, sua capacidade de deformação. Assim, os teores mais altos de HTPB nas formulações levaram à formação de membranas com maior resistência mecânica, característica imprescindível para tal aplicação.

Os dados referentes ao desempenho das membranas mostram que a presença de HTPB confere, de forma marcante, maior permeabilidade a gases nos poliuretanos ${ }^{[7]}$. Isso se deve ao caráter apolar desse segmento e à sua incapacidade de formação de ligações hidrogênio, em comparação ao segmento do poliéter $\mathrm{PPG}^{[7]}$. Dessa forma, há um decréscimo na miscibilidade entre os domínios rígidos e flexíveis em relação às amostras nas quais somente o poliéter está presente ${ }^{[7]}$. A maior segregação entre as fases provoca um aumento no volume livre entre as cadeias poliméricas favorecendo a permeabilidade dos gases. Isso se torna evidente quando o teor de segmentos flexíveis aumenta (razão $\mathrm{NCO} / \mathrm{OH}=1,5$ ).

Pode-se observar que os valores de permeabilidade a $\mathrm{CO}_{2}$ apresentaram diferenças significativas frente aos valores obtidos para $\mathrm{N}_{2}$. Observou-se que todas as membranas, indepen- dente da composição, apresentaram permeabilidade preferencial ao $\mathrm{CO}_{2}$. A permeabilidade em membranas geralmente aumenta com a redução do diâmetro cinético da molécula de gás, o que facilita a difusão das moléculas através das cadeias formadoras da membrana. Esse parâmetro é menor para o $\mathrm{CO}_{2}(d=3,30 \AA)$ do que para o $\mathrm{N}_{2}(d=3,64 \AA)^{[2,9]}$.

Comparando-se amostras de uma mesma razão, o aumento da quantidade de HTPB na formulação deu origem a membranas com maior permeabilidade ao $\mathrm{CO}_{2}$, ao passo que os valores desse parâmetro ficaram praticamente constantes quando $\mathrm{N}_{2}$ foi empregado como gás de purga. Conseqüentemente, a seletividade a $\mathrm{CO}_{2}$ em relação a $\mathrm{N}_{2}$ aumentou com o teor de HTPB na formulação, sendo esse aumento mais significativo para o percentual de $50 \%$. Nesse caso, à medida que aumentou o volume livre entre as cadeias, ocorreu um maior favorecimento para a difusão do gás.

As membranas obtidas a partir da razão $\mathrm{NCO} / \mathrm{OH}=3,0$, nas quais o teor de segmentos flexíveis é mais baixo, apresentaram comportamento semelhante, tanto na presença quanto na ausência de HTPB. No entanto, os valores de seletividade foram drasticamente reduzidos em comparação aos obtidos para maiores teores de segmentos flexíveis. Esses resultados ilustram como a presença de maiores proporções de segmentos rígidos desfavorece a separação entre os gases testados.

De uma forma geral, os resultados mostraram que, quanto maior foi o teor de segmentos provenientes do HTPB nas cadeias uretânicas, melhor foi o desempenho da membrana e que os resultados mais expressivos foram os obtidos com o maior teor desse poliol na formulação, tanto em termos de permeabilidade e de seletividade, quanto de resistência mecânica.

\section{Conclusões}

O acréscimo do teor de polibutadieno líquido hidroxilado (HTPB) nas cadeias uretânicas aumentou a permeabilidade 
das membranas a $\mathrm{CO}_{2} \mathrm{e}$ a seletividade a esse gás em relação ao $\mathrm{N}_{2}$. A presença de segmentos de HTPB aumentou a resistência mecânica do poliuretano e reduziu a sua elasticidade. Isso levou à obtenção de materiais mais resistentes para a manufatura de membranas.

As membranas uretânicas, formadas a partir de dispersões aquosas estudadas neste trabalho, apresentaram um bom desempenho além da vantagem de serem obtidas a partir de sistemas não-poluentes.

\section{Agradecimentos}

As autoras agradecem ao Conselho de Desenvolvimento Científico e Tecnológico (CNPq) e à Fundação de Amparo à Pesquisa do Estado do Rio de Janeiro (FAPERJ) pelo apoio financeiro.

\section{Referências Bibliográficas}

1. Hepburn, C. - "Polyurethane Elastomers", Elsevier, Amsterdam (1991).

2. Coutinho, F. M. B.; Delpech, M. C. \& Garcia, M. E. F. Polymer. Testing, 21, p.719 (2002).

3. Scharpman, F.; Couvercelle, J. P. \& Bunel, C. - Polymer, 39, p.965 (1998).

4. Haska, S. B.; Bayramli, E.; Pekel, F. \& Özkar, S. - Journal of Applied Polymer Science, 64, p.347, 1997.

5. Yang, J. M.; Lin, H. T. \& Lai, W. C. - Journal of Membrane Science, 208, p.105 (2002).

6. Huang, S. L. \& Lai, L. Y. - Journal of Applied Polymer Science, 58, p.1913 (1995).
7. Huang, S. L. \&. Lai L. Y. - Journal of Membrane Science, 105, p.137 (1995).

8. Wang, Z. F.; Wang, B.; Yang, Y. R. \& Hu C. P. - European Polymer Journal, 39, p. 2345 (2003).

9. Teo, L-S.; Chen, C-Y \& Kuo, J-F. - Journal of Membrane Science, 141, p. 91 (1998).

10. Cao, N.: Pegoraro, M.; Bianchi, F.; Di Landro, L. \& Zanderighi, J. - Journal of Applied Polymer Science, 48, p. 1831 (1993).

11. Chen, S. H.; Yua, K. C.; Houngb, S. L. \& Laic, J. Y. Journal of Membrane Science, 173, p.99 (2000).

12. Coutinho, F. M. B.; Delpech, M. C.; Alves, T. L. \& Gomes, A. S. - Polímeros: Ciência e Tecnologia, 12, p.284 (2002).

13. Coutinho, F. M. B.; Delpech, M. C. \& Alves, L. S. Polímeros: Ciência e Tecnologia, 10, p.40 (2000).

14. Coutinho, F. M. B. \& Delpech, M. C. - Polymer. Testing, 15, p.103 (1996).

15. Coutinho, F. M. B.; Delpech, M. C. \& Alves, L. S. Journal of Applied Polymer Science, 80, p. 566 (2001).

16. Coutinho, F. M. B. \& Delpech, M. C. - Polymer Degradation and Stability, 70, p. 49 (2000).

17. Boufi, S.; Gandini, A. \& Belgacem, M. N. - Polymer, 36, p. 1689 (1995).

18. Simek, L. \& Bohdanecky, M. - Journal of Applied Polymer Science, 58, p. 897 (1995).

19. Chen, Tk.; Hwung, C. J. \& Hou, C. C. - Polymer and Engineering Science, 32, p.115 (1992).

Enviado: $12 / 05 / 04$ Aprovado: 13/08/04 